\title{
Fatores motivacionais de idosos praticantes de exercícios físicos: um estudo baseado na teoria da autodeterminação
}

\author{
Simone Teresinha Meurer \\ Tânia Rosane Bertoldo Benedetti \\ Universidade Federal de Santa Catarina \\ Giovana Zarpellon Mazo \\ Universidade do Estado de Santa Catarina
}

\begin{abstract}
Resumo
Objetivou-se analisar os fatores e índices motivacionais de idosos participantes de um programa de exercícios físicos e a sua relação com o tempo de participação. Participaram 140 idosos. Para a coleta dos dados utilizouse o Inventário de Motivação para a Prática Regular de Atividades Físicas, duas perguntas abertas sobre o motivo de adesão e permanência no referido programa e ficha de identificação do idoso. Os dados foram analisados com auxílio da estatística descritiva e o teste de Mann-Whitney $(p<0,05)$. Verificou-se que a recomendação médica e o prazer foram os principais fatores de permanência no programa. O tempo de prática mostrou-se um fator diferencial para a mudança nas causas da motivação, possivelmente advindas da satisfação das necessidades psicológicas básicas. Destaca-se a importância de conhecer os fatores motivacionais de idosos participantes dos programas de exercícios físicos bem como da realização de atividades que auxiliem no desenvolvimento de aspectos da motivação intrínseca.
\end{abstract}

Palavras-chave: motivação; exercícios físicos; idoso.

\begin{abstract}
Motivational factors in aged practitioners of physical exercises: a study based on the theory of selfdetermination. The objective was to analyze the motivational factors and indices of elderly participants in an exercise program and its relationship to the time of participation. 140 elderly participated in the program. To collect data we used the Motivation Inventory Practice for Regular Physical Activity, two open questions about the reason of membership and permanence in that programme and identification form for the elderly. The data were analyzed using descriptive statistics and Mann-Whitney test $(p<0.05)$. It was found that the doctor's recommendation and pleasure were the main factors to remain in the program. The practice time proved to be a differentiating factor for change in the causes of motivation, possibly arising from the satisfaction of basic psychological needs. We emphasize the importance of knowing the motivational factors of elderly participants of exercise programs and carrying out activities that assist in developing aspects of intrinsic motivation.
\end{abstract}

Keywords: motivation; physical exercises; older adults.

\section{Introdução}

A Teoria da Autodeterminação (TAD) vem sendo utilizada em estudos (Wilson, Mack, \& Grattan, 2008, Wilson, Rodgers, Blanchard, \& Gessel, 2003) para explicar os diferentes fatores motivacionais que levam a população a iniciar, persistir e/ou abandonar a prática de atividades físico-esportivas. A motivação, nessa teoria, é compreendida dentro de um continuum e pode variar desde a sua forma mais autônoma (autodeterminada), até aquela controlada por aspectos externos (Edmunds, Ntoumanis, \& Duda, 2006), considerando que as regulações externas manifestam-se de diferentes formas (Ryan \& Deci, 2000). Assim, o continuum proposto pela TAD inclui a motivação intrínseca, extrínseca e amotivação (Ntoumanis, 2005).

Há indicativos de que quando os fatores de motivação são de origem intrínseca são mais significativos para a permanência das pessoas na prática de exercícios físicos (Reed \& Cox, 2007; Ryan, Frederick, Lepes, Rubio, \& Sheldon, 1997; Standage, Sebire, \& Loney, 2008). Estudos (Brickell \& Chatzisarantis, 2007, Edmunds et al. 2006, Wilson et al., 2003) também apontam que pessoas motivadas por fatores extrínsecos, porém com regulações intrínsecas para a prática físico-esportiva, apresentaram maior adesão a essas práticas.

Para o desenvolvimento da motivação intrínseca, o ser humano precisa ter satisfeitas as necessidades psicológicas básicas (autonomia, competência e relações sociais) (Mcdonough 
\& Crocker, 2007) e o tempo de participação em exercícios físicos também pode interferir sobre as necessidades psicológicas básicas e modificar o lócus de causa para a prática de exercícios físicos. Assim, se o indivíduo permanece engajado em algum programa por um tempo elevado, é possível que ocorram mudanças em relação ao tipo de motivação, podendo ser mais autodeterminado (Murcia, Gimeno, \& Coll, 2007, Wilson et al., 2003).

Embora a prática de exercícios físicos não possa interromper o processo biológico do envelhecimento, estudos evidenciam que o exercício físico pode minimizar efeitos fisiológicos e a progressão de doenças crônicas (Department of Health and Human Services [DHHS], 2008, Gillespie et al., 2003), comuns nesta fase da vida. Há também evidências de benefícios psicológicos e cognitivos para idosos, resultantes de sua participação em exercícios físicos (Borges, 2009; ChodzkoZajko et al., 2009, Mather et al., 2002).

Diante dos ganhos decorrentes da prática de exercícios físicos apontados, torna-se importante conhecer aspectos que podem influenciar os idosos na adesão e na manutenção de um estilo de vida ativo e saudável. Desta forma, evidencia-se a importância de conhecer os fatores de motivação e assim propor intervenções para uma mudança de comportamento.

Assim, o objetivo do presente estudo foi analisar os fatores e índices motivacionais de idosos participantes de um programa de exercícios físicos e a sua relação com o tempo de participação.

\section{Materiais e métodos}

Esta pesquisa foi aprovada pelo Comitê de Ética de Seres Humanos da Universidade Federal de Santa Catarina, projeto $n^{\circ} 078 / 2008$.

Os idosos foram informados sobre a pesquisa e assinaram o Termo de Consentimento Livre e Esclarecido em duas vias, ficando uma de posse dos idosos e outra dos pesquisadores.

A população do estudo foi de 209 idosos cadastrados no Programa Floripa Ativa - Fase B. A fase B do programa Floripa Ativa tem como principal objetivo a prevenção secundária e terciária e atende idosos com comorbidades leves a moderadas e aqueles já reabilitados na Fase A. Consiste em aulas de ginástica realizadas 3 vezes por semana, com duração de 60 minutos a sessão, enfatizando as diferentes qualidades físicas, principalmente, força, equilíbrio, flexibilidade, coordenação e resistência aeróbia.

Os critérios de elegibilidade para a participação no estudo foram: disponibilidade em participar da pesquisa; ter 60 anos de idade ou mais; responder os questionários de motivação para a prática de atividades físicas; responder as questões abertas sobre motivo de adesão e permanência no programa de exercícios físicos e participar do programa de forma assídua (verificado por meio das listas de frequência, sendo necessária presença em $75 \%$ das aulas no período de agosto a dezembro de 2008).

Assim, participaram do estudo 140 idosos, sendo 116 do sexo feminino e 24 do masculino. A idade variou entre 60 a 88 anos.

Como instrumento para a coleta de dados foi utilizado o Inventário de Motivação para a Prática Regular de Atividades
Físicas (IMPRAF - 54), que tem como pressuposto teórico a TAD e identifica os fatores de motivação em relação ao tempo presente. Ele foi construído e validado por Balbinotti e Barbosa (2006) para diferentes faixas etárias no Brasil, desde adolescentes (13 anos) a idosos (83 anos).

O IMPRAF - 54 é constituído por 54 itens, agrupados em 9 blocos, que são, de forma individual, avaliados por meio de uma escala Likert de 5 pontos, desde "isso me motiva pouquíssimo" a "isso me motiva muitíssimo" (Anexo 1).

Assim, esse instrumento mede 6 fatores associados à motivação para a prática regular de atividades físicas: controle de estresse, saúde, sociabilidade, competitividade, estética e prazer. O coeficiente Alpha para as dimensões da escala foi: Controle do Estresse $(\alpha=0,89)$, Saúde $(\alpha=0,87)$, Sociabilidade ( $\alpha=0,94)$, Competitividade $(\alpha=0,94)$, Estética $(\alpha=0,91)$ e Prazer $(\alpha=0,82)$.

Por meio desta escala, são identificados os fatores que são considerados mais motivadores para a prática de atividade física. Para contextualizar os resultados, são utilizadas tabelas normativas, fornecidas no manual de aplicação do instrumento, sendo que os escores brutos são transformados em percentis, que permitem comparar o desempenho do avaliado com seu grupo, conforme sexo e idade. De acordo com os percentis, foram criadas duas categorias, "motivação alta" e "motivação baixa". Percentis acima de 60 foram classificados como "motivação alta".

Associado a este questionário, foram utilizadas duas perguntas abertas sobre os motivos de ingresso e permanência no programa: "Qual foi o motivo pelo qual o senhor (a) ingressou no programa de exercícios físicos?" e "Por qual(is) motivos o senhor (a) permanece no programa de exercícios físicos?".

As informações em relação ao tempo de participação no programa de exercícios físicos foram obtidas a partir da ficha de identificação do idoso. Foram criadas duas categorias $(>$ de um ano e $\leq$ a um ano de prática), considerando os indicativos da literatura que evidenciam modificações psicológicas após um ano de prática de exercícios físicos (Borges, 2009).

A coleta de dados foi realizada por profissionais de Educação Física e acadêmicos bolsistas, devidamente treinados, no local onde as aulas são ministradas. Para a aplicação do questionário e da entrevista semi-estruturada (ambos em forma de entrevista individual), foram agendados horários antes e após as aulas.

Os dados foram analisados com o auxílio do programa SPSS ${ }^{\circledR}$ for Windows 15.0. Utilizou-se estatística descritiva com as medidas de tendência central, dispersão, frequência e tabelas de contingência. Para verificar a diferença entre índices de motivação e tempo de prática foi utilizado o teste de MannWhitney. O nível de significância adotado foi de 5\%.

\section{Resultados}

Neste estudo, dos 140 idosos praticantes de exercícios físicos, 116 são do sexo feminino e 24 do masculino; 61 participam do programa há mais de um ano e 79 há menos de um ano. A média de idade do grupo é de 67,54 anos $(D P=6,6)$.

Ao agruparmos as respostas obtidas nas perguntas abertas em relação ao fator de motivação para inserção e permanência 
no programa de exercícios físicos, foi possível estabelecer 5 categorias de análise, apresentadas na Tabela 1.

A recomendação médica e a saúde foram os principais fatores para a inserção dos idosos no programa de exercícios físicos e, como fatores de permanência, a recomendação médica e o prazer foram os fatores com maiores pontuações.

Os fatores e índices de motivação mostram a motivação atual de permanência no programa. Por meio dos escores obtidos para cada fator, apresentam-se as médias. Os escores foram classificados em duas categorias (Motivação Alta e Motivação Baixa).

Evidenciou-se que a saúde, o prazer e a sociabilidade são os fatores de motivação que obtiveram as maiores médias. E, em relação aos índices, identificou-se que para todos os fatores,

Tabela 1

Motivação e permanência de idosos em um programa de exercícios físicos.

\begin{tabular}{lcccc}
\hline & Fatores de inserção & & \multicolumn{2}{c}{ Fatores de Permanência } \\
& F & $\mathbf{\%}$ & F & \% \\
\hline Recomendação médica & 76 & 48,6 & 53 & 37,8 \\
Saúde & 39 & 33,4 & 19 & 13,5 \\
Prazer & 4 & 2,9 & 45 & 32,1 \\
Socialização & 12 & 8,6 & 19 & 13,6 \\
Acompanhar alguém & 9 & 6,4 & 4 & 2,9 \\
\hline
\end{tabular}

Tabela 2

Escores de classificação da motivação

\begin{tabular}{lccc}
\hline & Média $(\boldsymbol{D P})$ & Motivação alta (\%) & Motivação baixa (\%) \\
\hline Controle de Estresse & $30,46(7,41)$ & 69,3 & 30,7 \\
Sociabilidade & $35,06(6,05)$ & 76,4 & 23,6 \\
Saúde & $37,15(3,71)$ & 70 & 30 \\
Competitividade & $17,19(9,32)$ & 72,9 & 27,1 \\
Estética & $26,36(9,00)$ & 44,3 & 55,7 \\
Prazer & $35,56(5,38)$ & 57,1 & 42,9 \\
\hline
\end{tabular}

exceto para a estética, a maioria dos idosos tem motivação alta.

$\mathrm{Na}$ Tabela 3, pode-se observar a distribuição percentual dos idosos em relação aos fatores e índices de motivação e tempo de inserção no programa de exercícios físicos. Em relação aos idosos praticantes há mais de um ano, evidencia-se motivação elevada pelos 3 fatores destacados (sociabilidade, saúde e prazer) e observa-se que, quando comparados aos que praticavam exercícios físicos há menos de um ano, o percentual de idosos na categoria "motivação alta" para o fator prazer foi maior.

Quando realizada a comparação entre os fatores e os grupos ( $>$ de um ano e $\leq$ há um ano de prática), foi identificada diferença estatisticamente significativa no que se refere ao prazer pela prática, tendo escores superiores os praticantes há mais de um ano.

Tabela 3

Tempo de participação (TP) no programa e classificação dos idosos de acordo com os índices e fatores de motivação (IF Motivação) para a prática de exercícios físicos

\begin{tabular}{|c|c|c|c|c|c|c|c|}
\hline IF $T$ & \multicolumn{2}{|c|}{$\leq$ há um ano } & \multirow{2}{*}{$\begin{array}{c}\begin{array}{c}\text { Mann- } \\
\text { Whitney }\end{array} \\
\text { Rank }\end{array}$} & \multicolumn{2}{|c|}{$>\underset{(\%)}{>\text { de um ano }}$} & \multirow{2}{*}{$\begin{array}{c}\text { Mann- } \\
\text { Whitney } \\
\text { Rank }\end{array}$} & \multirow[t]{2}{*}{$\begin{array}{c}p \\
\text { valor }\end{array}$} \\
\hline Motivação & Alta & Baixa & & Alta & Baixa & & \\
\hline Saúde & 72,2 & 27,8 & 70,11 & 67,2 & 32,8 & 71,00 & 0,89 \\
\hline Sociabilidade & 74,7 & 25,3 & 67,47 & 78,7 & 21,3 & 74,43 & 0,30 \\
\hline Prazer* & 51,9 & 48,1 & 64,01 & 63,9 & 36,1 & 78,1 & $0,02 *$ \\
\hline
\end{tabular}

* No fator prazer houve diferenças entre os grupos $(\mathrm{U}=1896,5 ; p=0,02)$.

\section{Discussão}

O presente estudo identificou que a recomendação médica e o prazer foram os fatores responsáveis pela permanência de idosos no programa de exercícios físicos. Além disso, a saúde e a sociabilidades são fatores que muito motivam os idosos a participar do programa de exercícios físicos.

Em relação aos índices de motivação, todos os fatores foram classificados como sendo de motivação alta, exceto a estética. Entende-se que a estética é um fator de motivação cuja importância diminui com a idade, uma vez que a preocupação centra-se em aspectos relacionados à sensação de bem estar e saúde, ao invés da aparência.

A competitividade foi o fator de motivação que apresentou baixa média, porém, ao lançar os valores nas tabelas normativas, de acordo com o sexo e idade, as pontuações foram classificadas, em sua maioria, como sendo de motivação alta. Isso se explica pelo fato de os idosos que participaram do estudo que estabeleceu as tabelas normativas, terem pontuações praticamente nulas nesse fator. 
Os idosos participantes do Programa Floripa Ativa - Fase B têm sua iniciação no programa após consulta médica e, um dos critérios para a inclusão no programa é a presença de uma comorbidade leve ou moderada. Isto pode ter influenciado no fato da maioria dos idosos terem a recomendação médica como o principal motivo de ingresso no programa, bem como na elevada média obtida pelo fator saúde.

De forma idêntica aos resultados encontrados no presente estudo, Stiggelbout, Hopman-Rock e Mechelen (2008) destacaram a melhoria da saúde como um constructo motivacional para idosos participantes de programas de exercícios físicos. Também Cohen-Mansfield, Marx, Biddison, e Guralnik (2004) analisaram 324 idosos com idades entre 74 e 85 anos, concluindo que, para quase $70 \%$ dos participantes, o aconselhamento médico para a prática de exercício físico, dentre outras, influenciaram a adesão ao exercício físico.

Analisando a motivação pelo fator saúde de acordo com o contexto estudado, percebe-se que este tem uma regulação extrínseca, ou seja, tal comportamento é realizado para suprir uma demanda externa, a indicação do médico. Destaca-se o estudo de Wilson et al. (2003) que aponta que esse fator motivacional pode assumir uma regulação identificada ou integrada quando o indivíduo passa a considerá-lo importante e aprecia os resultados e os benefícios da participação em tal atividade.

Quando os idosos foram questionados sobre os motivos que os mantêm participando do programa de exercícios físicos, a recomendação médica manteve o maior percentual de respostas. Porém, observou-se um aumento de 29,2\% das respostas em relação ao fator prazer pela prática, o que evidencia que gostar da atividade realizada é um fator importante para a manutenção dentro do programa de exercícios físicos. O prazer pela prática também foi um dos fatores apontados como motivo para a participação de 2.020 idosos em diferentes programas de exercícios físicos (Stiggelbout et al., 2008).

A elevada pontuação recebida pelo fator prazer e o aumento na frequência desta categoria como motivo de permanência na prática de exercícios físicos pelos idosos é um dado interessante. Considerando os pressupostos teóricos da TAD, o prazer pela prática pode ser interpretado como motivação intrínseca. A motivação intrínseca supõe o compromisso de uma pessoa com a atividade por causa do prazer e pelo desfrutar que esta produz; a atividade tem um fim em si mesma (Ryan \& Deci, 2000).

De acordo com Murcia et al. (2007), se o indivíduo permanece engajado em algum programa por um tempo elevado, é possível que ocorram mudanças em relação ao local de causalidade dos fatores motivacionais para a permanência na prática de exercícios físicos. Este fato parece que vem acontecendo com os idosos inseridos há mais de um ano no Programa Floripa Ativa - Fase B. Ao comparar os escores brutos de motivação (fatores saúde, prazer e sociabilidade) entre os idosos praticantes há um ano ou menos e aqueles praticantes há um ano ou mais, foi encontrada diferença estatisticamente significante no fator prazer.

Estudos evidenciam que quanto maior o tempo de prática, maior o nível de motivação por aspectos intrínsecos. Wilson et al. (2003) realizaram um estudo experimental que verificou as alterações longitudinais nos níveis de motivação de praticantes de programas de exercícios físicos. Os autores observaram aumento nos níveis de regulação identificada e motivação intrínseca, o que demonstra que quanto mais tempo se pratica exercício físico sistematizado, menor a probabilidade de desistência, pois mais motivado intrinsecamente estará o indivíduo. Resultados semelhantes foram encontrados por Murcia et al. (2007), que também concluíram que um maior tempo de prática está relacionado com o maior índice de autodeterminação.

Neste sentido, a TAD explica que para a autodeterminação ser desenvolvida, há necessidade de o indivíduo ter satisfeito as necessidades psicológicas básicas (autonomia, competência, relacionamento social) (Gagne, Ryan, \& Bargmann, 2003, Reiss, Sheldon, Gable, Roscoe, \& Ryan, 2000). A autonomia reflete o desejo de participar de atividades em que a possibilidade de escolha na realização do comportamento esteja presente (Ryan \& Deci, 2000). A necessidade de competência está ligada a sentir-se capacitado e confiante para realizar um determinado comportamento com determinada aptidão. Quanto ao relacionamento social, trata-se da necessidade de perceber que o comportamento é reconhecido positivamente por outras pessoas, ou que a prática facilita a socialização (Ingledew, Markland, \& Sheppard, 2004).

Há indicativos de que os idosos que praticaram exercícios físicos há mais de um ano desenvolveram aspectos relacionados à motivação autônoma (prazer pela prática), sendo que entre os aspectos explicativos pode-se apontar o aumento da percepção de competência, bem como a satisfação da necessidade de relacionamento social proporcionada pelos idosos participantes do programa.

Wilson e Rodgers (2002) afirmam que quando o contexto social é adequado para o desenvolvimento das necessidades psicológicas básicas, haverá maior facilidade para o desenvolvimento da autodeterminação. Assim, o programa de exercícios físicos Floripa Ativa - Fase B parece ser um ambiente favorável para o desenvolvimento de fatores autônomos de participação em exercícios físicos.

Poucos estudos nacionais analisaram as necessidades psicológicas básicas em idosos e sua associação com o comportamento em relação ao exercício físico, bem como não encontramos instrumentos validados para tal no contexto brasileiro. Desta forma, há limitações para realizar comparações dos resultados. Ainda, pelas características específicas da amostra, não podem ser realizadas generalizações dos resultados.

\section{Conclusão}

Considerando os resultados identificados no presente estudo, parece que a recomendação médica associada ao prazer na realização da prática foram fatores de grande importância para a permanência de idosos no programa de exercícios físicos Floripa Ativa - Fase B.

Evidencia-se, pela teoria da autodeterminação, que após um ano de manutenção dos idosos no programa de exercícios físicos, ocorrem mudanças importantes na causa da motivação, possivelmente advindas da satisfação das necessidades psicológicas básicas desenvolvidas no programa de exercícios 
físicos.

Destaca-se a importância de conhecer os fatores motivacionais de idosos participantes dos programas de exercícios físicos bem como da realização de atividades que auxiliem no desenvolvimento de aspectos da motivação intrínseca.

\section{Referências}

Balbinotti, M. A. A., \& Barbosa, M. L. L. (2006). Manual de aplicação do inventário motivação à prática regular de atividades fisicas (IMPRAF - 126). Universidade Federal do Rio Grande do Sul: Porto Alegre.

Borges, L. J. (2009). Influência de um programa de exercício físico na saúde mental e na aptidão funcional de idosos usuários dos Centros de Saúde de Florianópolis. (Dissertação de Mestrado). Universidade Federal de Santa Catarina, Florianópolis.

Brickell, T. A., \& Chatzisarantis, N. L. D. (2007). Using self-determination theory to examine the motivational correlates and predictive utility of spontaneous exercise implementation intentions. Psychology of Sport and Exercise, 8(5), 758-770.

Chodzko-Zajko, W. J., Proctor, D. N., Singh, M. A. F., Minson, C. T., Nigg, C. R., Salem, G. J, \& Skinner, J. S (2009). Exercise and physical activity for older adults. Medicine and Science in Sports and Exercise, 4l(7), 1510-1530.

Cohen-Mansfield, J., Marx, M. S., Biddison, J. R., \& Guralnik, J. M. (2004) Socio-environmental exercise preferences among older adults. Preventive Medicine, 38(6), 804-811.

Department of Health and Human Services (DHHS) (2008). Physical Activity Guidelines Advisory Committee Report. Rockville (MD): U.S.

Edmunds, J., Ntoumanis, N., \& Duda, J. L. (2006). A Test of self-determination theory in the exercise domain. Journal of Applied Social Psychology, 36(9), 2240-2265.

Gagne, M., Ryan, R. M., \& Bargmann, K. (2003). Autonomy support and need satisfaction in the motivation and well-being of gymnasts. Journal of Applied Sport Psychology, 15(4), 372-390.

Gillespie, L. D., Gillespie, W. J., Robertson, M. C., Lamb, S. E., Cumming, R. G., \& Rowe, B. H. (2003). Interventions for preventing falls in elderly people. Cochrane Database of Systematic Reviews, 4, CD000340.

Ingledew, D. K., Markland, D., \& Sheppard, K. E. (2004). Personality and selfdetermination of exercise behavior. Personality and Individual Differences, 36(8), 1921-1932.

Mather, A. S., Rodriguez, C., Guthrie, M. F., Mcharg, A. M., Reid, I. C., \&
Mcmurdo, M. E. (2002). Effects of exercise on depressive symptoms in older adults with poorly responsive depressive disorder: randomized controlled trial. British Journal of Psychiatry, 180(6), 411-415.

McDonough, M., \& Crocker, P. (2007). Testing self-determined motivation as a mediator of the relationship between psychological needs and affective and behavioral outcomes. Journal of Sport \& Exercise Psychology, 29(5), 645-663.

Murcia, J. A. M., Gimeno, E. C., \& Coll, D. G. (2007). Analizando la motivación en el deporte: un estudio a través de la teoría de La autodeterminación. Apuntes de Psicología, 25(1), 35-51.

Ntoumanis, N. (2005). A Prospective study of participation in optional school physical education using a self-determination theory framework. Journal of Educational Psychology, 97(3), 444-453.

Reed, C., \& Cox, R. H. (2007). Motives and regulatory style underlying senior athletes' participation in sport. Journal of Sport Behavior, 30(3), 307 - 329.

Reiss, H. T, Sheldon, K. M, Gable, S. L., Roscoe, J. \& Ryan, M. R. (2000). Daily well-being: the role of autonomy, competence, and relatedness. Personality and Social Psychology Bulletin, 26(4), 419-435.

Ryan, R. M., \& Deci, E. L. (2000). Intrinsic and extrinsic motivations: classic definitions and new directions. Contemporary Educational Psychology, 25(1), 54-67.

Ryan, R.M., Frederick, C.M., Lepes, D., Rubio, N., \& Sheldon, M. (1997) Intrinsic motivation and exercise adherence. International Journal of Sport Psychology, 28(4), 335-354.

Standage, M., Sebire, S.J., \& Loney, T. (2008). Does exercise motivation predict engagement in objectively assessed bouts of moderate-intensity exercise? A self-determination theory perspective. Journal of Sport \& Exercise Psychology, 30(4), 337-352.

Stiggelbout, M., Hopman-Rock, M., \& Mechelen, W. (2008). Entry correlates and motivations of older adults participating in organized exercise programs. Journal of Aging and Physical Activity, 16(3), 342-354.

Wilson, P. M., Mack, D. E., \& Grattan, K. P. (2008). Understanding motivation for exercise: a self-determination theory perspective. Canadian Psychology, 49(3), 250-256.

Wilson, P. M., \& Rodgers, W. M. (2002). The relationships between exercise motives and physical self-esteem in female exercise participants: an application of self-determination theory. Journal of Applied Biobehavioral Research, 7(1), 30-43.

Wilson, P. M., Rodgers, W. M., Blanchard, C. M., \& Gessel, J. (2003). The relationships between physiological needs, self determined motivation, exercise attitudes and physical fitness. Journal of Applied Social Psychology, 33(11), $2373-2397$.

Simone Teresinha Meurer, mestre em Educação Física pela Universidade Federal de Santa Catarina, doutoranda na Universidade Federal de Santa Catarina. Endereço para correspondência: Núcleo de Cineantropometria e Desempenho Humano, Campus Universitário, Trindade. CEP: 88040-900. Telefone: (48) 3721-8562. E-mail: simonemeurer@yahoo.com.br

Tânia Rosane Bertoldo Benedetti, pós doutora em Kinesiology and Community Health pela University of Illinois at Urbana e doutora em Enfermagem, é professora do Departamento de Educacao Física da Universidade Federal de Santa Catarina. E-mail: benedetti@cds.ufsc.br Giovana Zarpellon Mazo, doutora em Ciência do Desporto pela Universidade do Porto, é professora na Universidade do Estado de Santa Catarina. E-mail: d2gzm@udesc.br 